\title{
ALCANCES Y LIMITACIONES EN LA MEDICIÓN DE PELÍCULAS CON UN DIFRACTÓMETRO CONVENCIONAL: BRUKER D8 ADVANCE
}

\author{
Scope and limitations on the measurement of movies with \\ a conventional diffractometer: Bruker D8 ADVANCE
}

\section{EPISTEMUS}

ISSN: 2007-8196 (electrónico)

ISSN: 2007-4530 (impresa)

\section{Abraham Mendoza Córdova ${ }^{1}$ \\ Ramón Ochoa Landín² \\ Mario Flores Acosta $^{3}$}

Recibido: 23 de junio de 2016,

Aceptado: 30 de noviembre de 2016

Autor de Correspondencia:

M.C. Abraham Mendoza Córdova

Correo: mendozacordova@ciencias.uson.mx

\section{Resumen}

Las películas delgadas son capas de materiales con espesores que van desde algunos cuantos nanómetros hasta algunos micrómetros, dichas capas son creadas por técnicas tanto físicas como químicas. Para la caracterización estructural de los materiales cristalinos en forma de películas es indispensable la técnica de difracción de rayos X (DRX). El difractómetro indicado para hacer las mediciones debe de incluir un accesorio conocido como espejo Gobel. En el presente trabajo se realizaron mediciones sobre películas de: $\mathrm{CdS}, \mathrm{AgOH}$ y $\mathrm{CdCO} 3$ utilizando un difractómetro convencional de polvos sin espejo Gobel, los resultados fueron favorables ya que se obtuvieron los patrones de difracción de los materiales, observándose una mejor definición en las películas de mayor espesor.

Palabras clave: Angulo rasante, películas delgadas, difractómetro convencional.

\begin{abstract}
The thin films are materials with layers, those can vary in thickness from nanometers to micrometers there are several ways to create this kind of layers by chemical and physical techniques. The x-ray diffraction technique is necessary to characterize the structural of crystalline materials as thin films. In order to realize the measurements the diffractometer needs to include the mirror known as Gobel. In this work we present some x-ray measurements of $\mathrm{CdS}, \mathrm{AgOH}$ and $\mathrm{CdCO} 3$ thin films. Those measurements were made using the conventional powder diffractometer without Gobel mirror, the results were similar because thin films diffraction patterns were obtained and we could observe a better definition in the thin films with major thickness.
\end{abstract}

Keywords: Thin films, conventional diffractometer, thickness.

1 Departamento de Geología, Universidad de Sonora/Correo: mendozacordova@ciencias.uson.mx

2 Departamento de Física, Universidad de Sonora/Correo: ramon.ochoa@correo.fisica.uson.mx

3 Departamento de Investigación en Física, Universidad de Sonora/Correo: mflores@cifus.uson.mx 


\section{INTRODUCCIÓN}

Entre las diversas utilidades que tienen las películas delgadas están: La producción de tratamientos interferenciales anti reflejantes, le fabricación de dispositivos ópticos. En la fabricación de celdas fotovoltaicas y detectores de luz, en la miniaturización de dispositivos optoelectrónicas. Se han desarrollado ya las fibras ópticas, las guías de onda y las válvulas ópticas en base a sistemas de películas delgadas y filamentos.

Dentro de las diferentes técnicas utilizadas para la caracterización de películas delgadas está la difracción de rayos $X$ (DRX). Dicha técnica es la indicada para realizar identificación de fases, cambios de fase, cálculo de tamaño de grano, constante de red y deformación de la red. En el caso de las películas delgadas la medición debe ser con ángulo rasante para eliminar la contribución de información relacionada al sustrato, que normalmente es un material amorfo como el vidrio [1]. El difractómetro para medir películas cuenta con un accesorio llamado espejo Gobel [2], dicho aditamento permite emitir sobre la película un haz paralelo de radiación (Figura 1) que suministra una alta densidad de rayos $X$, con lo cual se logra una mayor resolución en el patrón de difracción del material.

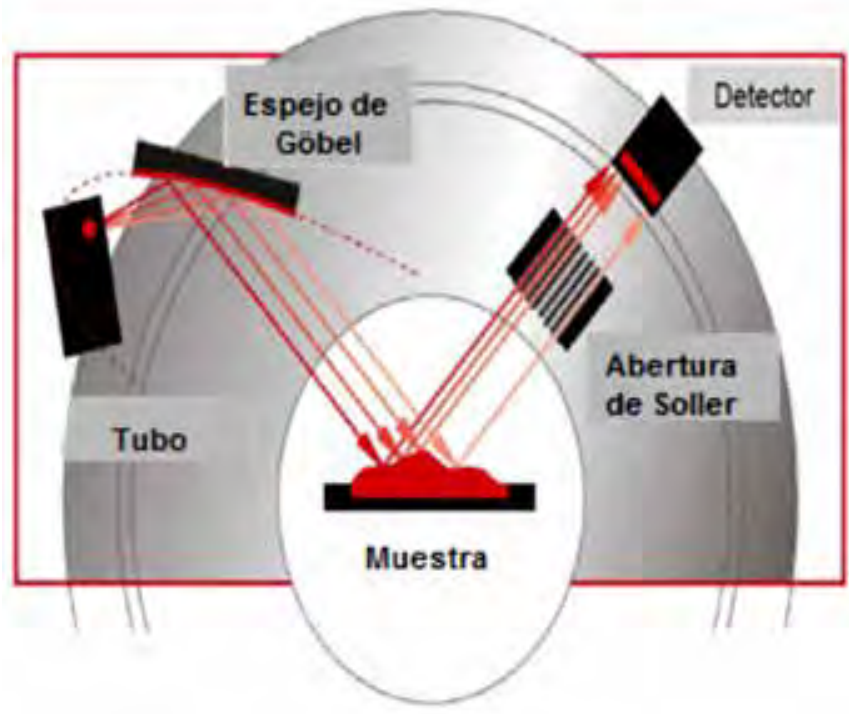

Figura 1. Esquema de la configuración con haz paralelo producido por el espejo Gobel, se utiliza para polvos y superficies irregulares con la configuración BraggBrentano $\Theta: 2 \Theta$, así como también para medir películas con haz rasante $2 \theta$ y $\theta$ fijo.

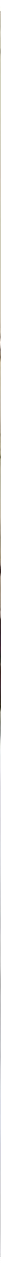




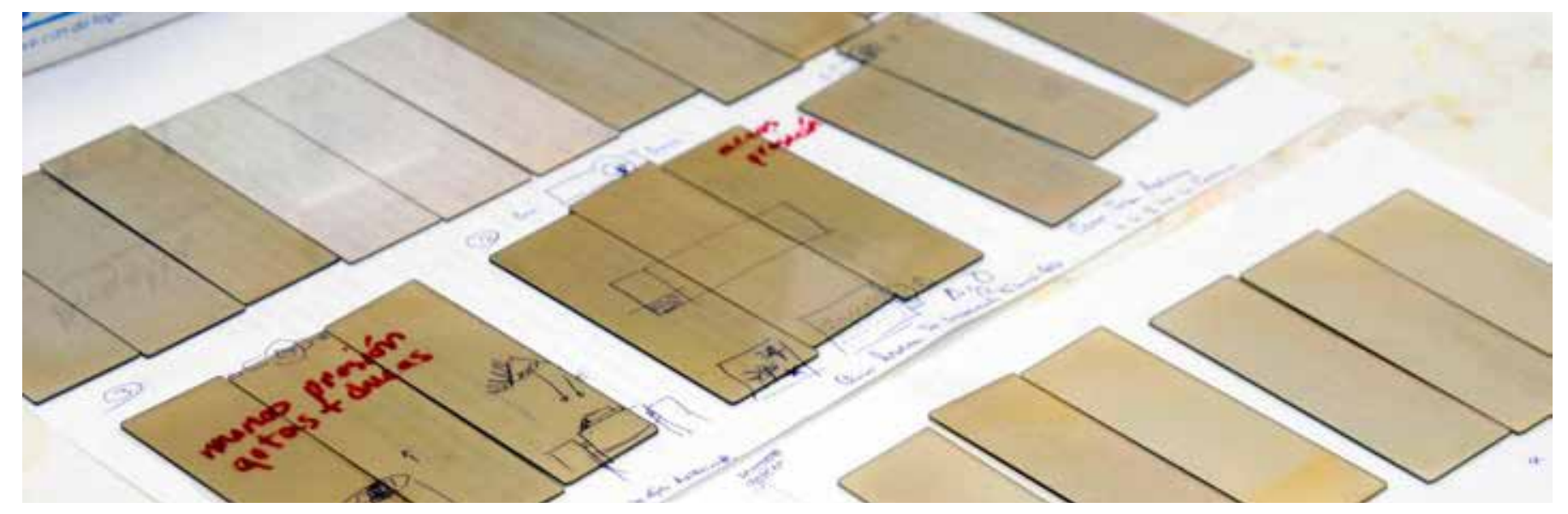

El equipo utilizado en este trabajo para hacer la medición de películas, particularmente de materiales semiconductores policristalinos como: $\mathrm{CdS}, \mathrm{AgOH}$, y $\mathrm{CdCO}_{3}$, es un difractómetro marca Bruker modelo D8 ADVANCE, con haz no paralelo utilizado para polvos y superficies pulidas en el esquema de la geometría BraggBrentano $\Theta: 2 \Theta$ (Figura 2). Este equipo no cuenta con el espejo Gobel pero puede medir con haz rasante utilizando el modo Detector Scan, con la configuración BraggBrentano solo $2 \theta$, donde $\theta$ se mantiene fijo.

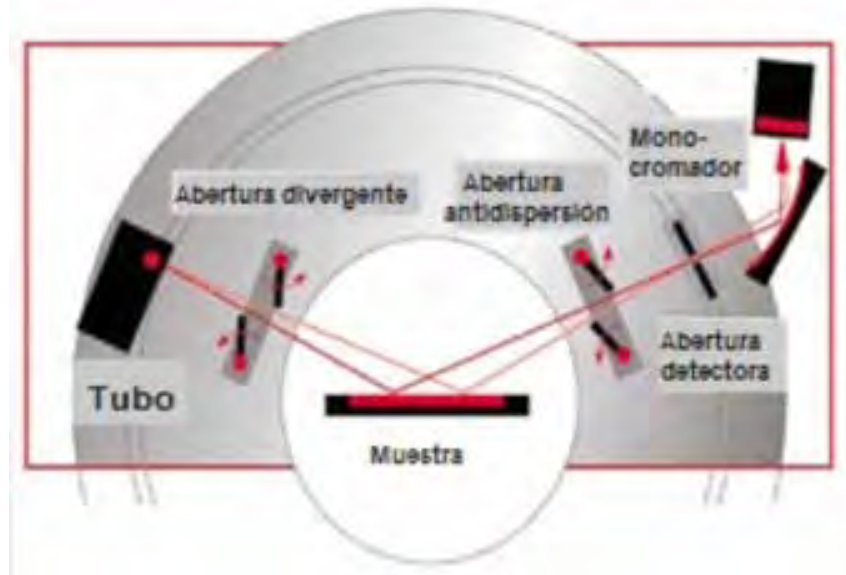

Figura 2. Esquema de la geometría Bragg-Brentano $\Theta: 2 \Theta$ con haz no paralelo utilizada para polvos $y$ superficies pulidas.

Para buscar una buena señal en las mediciones se experimentó con películas de diferentes espesores y una misma velocidad de escaneo ( $\mathrm{CdS}, \mathrm{AgOH})$, y con películas de un mismo espesor pero con diferente velocidad de escaneo $\left(\mathrm{CdCO}_{3}\right)$. Este experimento se hizo con la finalidad de determinar los parámetros con los que los materiales tuvieran la mejor respuesta de difracción.

\section{ELABORACIÓN DE PELÍCULAS}

Las películas estudiadas en este trabajo corresponden a materiales semiconductores consistentes en: CdS,
$\mathrm{AgOH}$ y $\mathrm{CdCO}_{3}$ los cuales se sintetizaron por el método de baño químico. Esta técnica consiste en adicionar en un reactor (Figura 3) una sal que contenga el ion metálico, un compuesto que compleje al ion metálico para evitar la precipitación del compuesto deseado, una sal que contenga al ion no metálico y un buffer para mantener el pH en la solución de reacción. En esta técnica, se parte de una solución acuosa de sales de los elementos de compuesto que se desea obtener. El sustrato de vidrio es sumergido en esta solución y a la vez la solución es sometida a calentamiento constante por un tiempo determinado, siendo estos los parámetros de depósito y los que proporcionan las propiedades de la película junto con las concentraciones de elementos en la solución. En la superficie del sustrato ocurre una reacción química provocando la formación de la película. Los compuestos que pueden ser depositados deben ser relativamente insolubles y químicamente estables en la solución y presentar una precipitación simple en una reacción iónica.

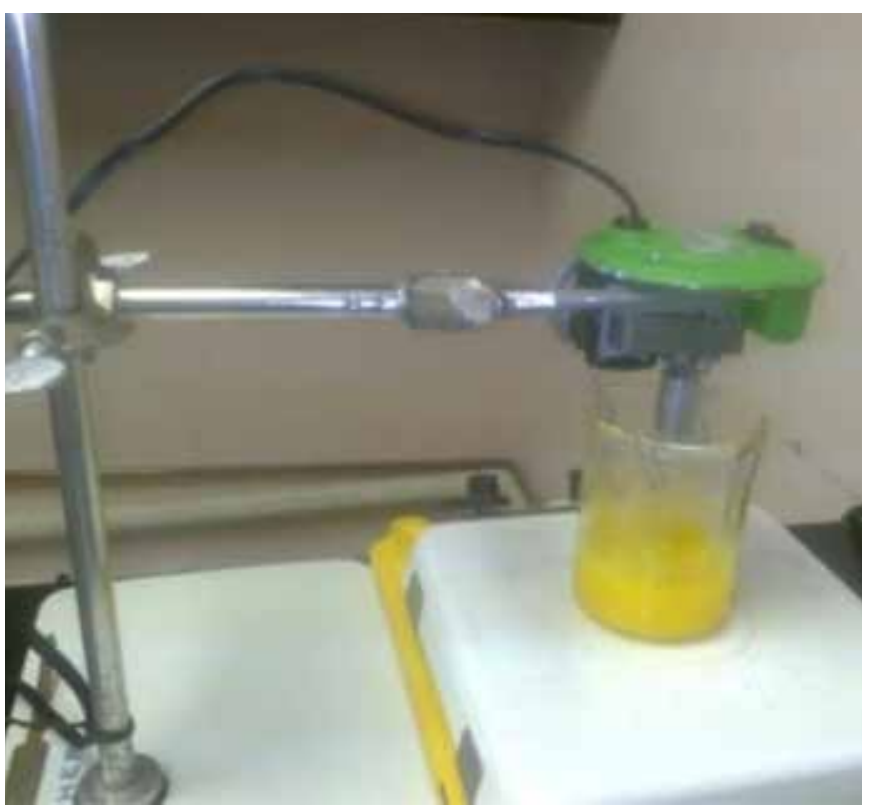

Figura 3. Equipo utilizado para realizar baño químico. 


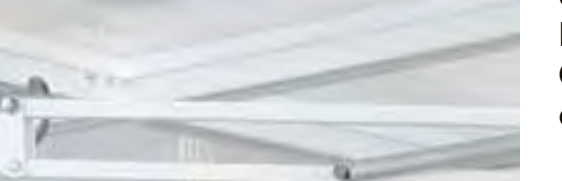

En la figura 3 se observa el equipo utilizado, conformado por un controlador de voltaje para la agitación mecánica de $16 \mathrm{rev} / \mathrm{min}$, con una determinada temperatura; en la figura se observa el buril diseñado y montado sobre motor de corriente directa con abertura de $2 \mathrm{~nm}$ donde se coloca el sustrato.

Para cada uno de los materiales sintetizados se utilizó su correspondiente agente complejante y precursor como reactivos [3], así como una determinada temperatura y pH. Los tiempos de deposición fueron cinco para el $\mathrm{CdS}$, cuatro para el $\mathrm{AgOH}$ y uno para el $\mathrm{CdCO}_{3}$ (Tabla 1). El espesor de las películas fue proporcional al tiempo de deposición, el cual físicamente se ve reflejado en el tono y en la textura de las muestras (Figura 4).

Tabla 1. Reactivos y condiciones para elaborar las películas de tres tipos de compuestos; el tiempo de deposición es determinante en el espesor que se obtiene.

\begin{tabular}{|c|c|c|c|c|c|c|}
\hline MATERIAL & PRECURSOR & $\begin{array}{c}\text { AGENTE } \\
\text { COMPLEJANTE }\end{array}$ & $\mathrm{pH}$ & $\mathrm{T}\left({ }^{\circ} \mathrm{C}\right)$ & $\begin{array}{l}\text { TIEMPO DE } \\
\text { DEPOSICIÓN } \\
(\text { min) }\end{array}$ & $\begin{array}{l}\text { ESPESOR } \\
\quad(\mathrm{nm})\end{array}$ \\
\hline \multirow{5}{*}{$\mathrm{CdS}$} & \multirow{5}{*}{$\begin{array}{c}\mathrm{CdCl} 2 \\
\mathrm{KOH} \\
\mathrm{CH} 4 \mathrm{~N} 2 \mathrm{~S} \\
\text { (Tiourea) }\end{array}$} & \multirow{5}{*}{$\begin{array}{c}\mathrm{Na} 3 \mathrm{C} 6 \mathrm{H} 5 \mathrm{O} 7 \\
\text { (Citrato de sodio) }\end{array}$} & \multirow[t]{5}{*}{10} & \multirow[t]{5}{*}{70} & 20 & 40 \\
\hline & & & & & 25 & 60 \\
\hline & & & & & 30 & 70 \\
\hline & & & & & 45 & 85 \\
\hline & & & & & 60 & 100 \\
\hline \multirow{4}{*}{$\mathrm{AgOH}$} & \multirow{4}{*}{$\begin{array}{c}\mathrm{AgNO} 3 \\
\mathrm{KOH}\end{array}$} & \multirow{4}{*}{$\begin{array}{c}\mathrm{C} 6 \mathrm{H} 15 \mathrm{NO} 3 \\
\text { (Trietanolamina) }\end{array}$} & \multirow[t]{4}{*}{10} & \multirow[t]{4}{*}{25} & 30 & 110 \\
\hline & & & & & 60 & 140 \\
\hline & & & & & 90 & 180 \\
\hline & & & & & 120 & 210 \\
\hline $\mathrm{CdCO} 3$ & $\begin{array}{c}\mathrm{CdCl} 2 \\
\mathrm{NaHCO} 2\end{array}$ & 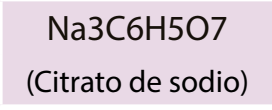 & 10 & 50 & 48 horas & $1 \mu \mathrm{m}$ \\
\hline
\end{tabular}

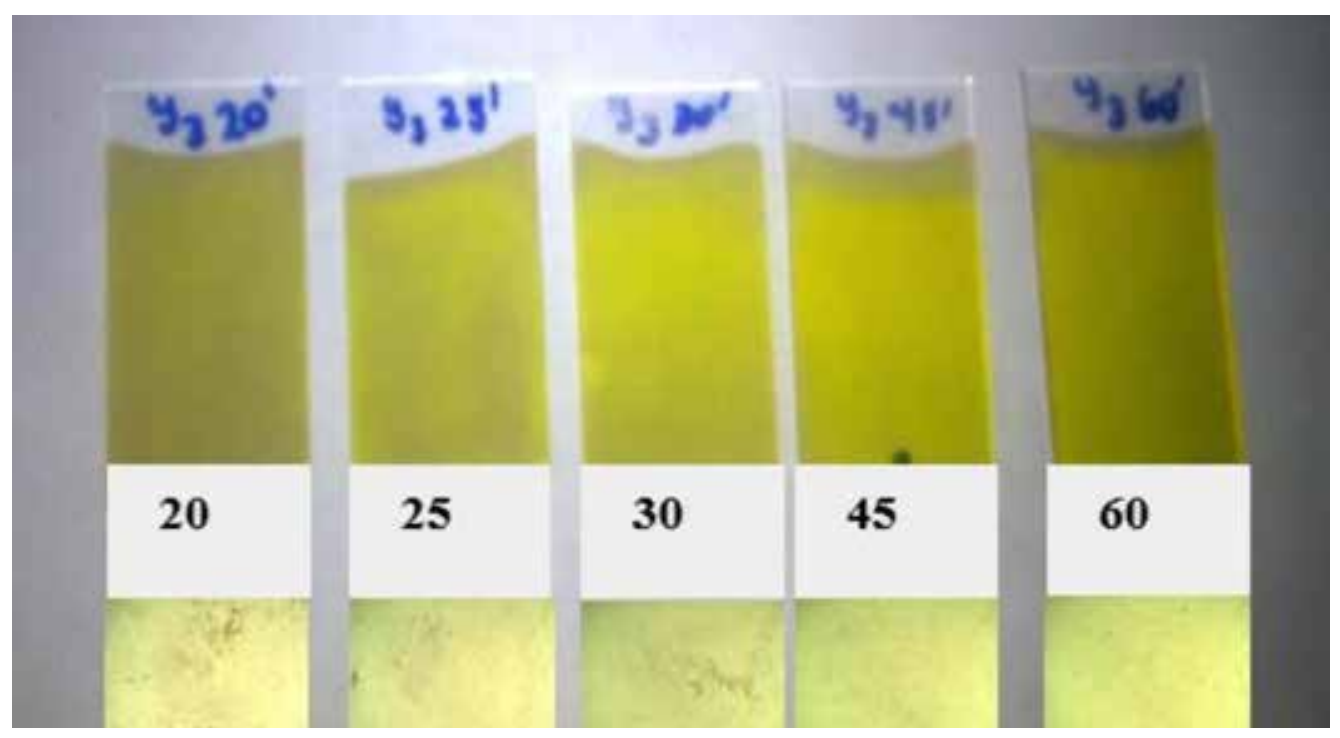

Figura 4. Películas CdS y tiempo de deposición.

En la figura 4 se aprecia en la parte superior de los números (Tiempo de deposición), las cinco películas de $\mathrm{CdS}$ en las que el único parámetro que cambia es el tiempo de deposición. El tono comienza con gris en la película de los veinte minutos y va cambiando a un color amarillo tenue que se intensifica conforme incrementa el tiempo. Las fotos de 
la parte inferior se tomaron con cámara digital integrada al microscopio en las cuales se deja ver la diferencia de textura.

\section{MEDICIÓN DE PELÍCULAS CON DRX}

Los parámetros de trabajo en el equipo fueron constantes para medir todas las películas de $\mathrm{CdS}$ y de $\mathrm{AgOH}$, mientras que en las mediciones de $\mathrm{CdCO}_{3}$ fue variable la velocidad de escaneo en una sola muestra (Tabla 2 ).

Tabla 2. Parámetros utilizados en la medición de películas con equipo convencional de polvo.

\begin{tabular}{|l|l|l|}
\hline Parámetros & $\mathrm{CdS}$ y $\mathrm{AgOH}$ & $\mathrm{CdCO}_{3}$ \\
\hline Intervalo & $32^{\circ}$ & $32^{\circ}$ \\
\hline Ángulo de incidencia & $3^{\circ}$ & $3^{\circ}$ \\
\hline Paso angular & $0.02^{\circ}$ & $0.02^{\circ}$ \\
\hline Velocidad de escaneo & $1 \mathrm{seg} /$ paso & $\begin{array}{l}1,2,3,4,5 \mathrm{seg} / \\
\text { paso }\end{array}$ \\
\hline Rendija & $1 \mathrm{~mm}$ & $1 \mathrm{~mm}$ \\
\hline $\mathrm{Kv}$, ma & 40,35 & 40,35 \\
\hline $\begin{array}{l}\text { Modo de escaneo } \\
\text { (Detector scan) }\end{array}$ & sólo 2 theta & sólo 2 theta \\
\hline
\end{tabular}

Para el caso del CdS y del AgOH se midieron cinco y cuatro películas respectivamente de diferente espesor, en intervalos de escaneo de $32^{\circ}$ y $82^{\circ}$ respectivamente. El tiempo de escaneo se probó con velocidades de 1, 2 y $3 \mathrm{seg} /$ paso. Los difractogramas que se presentan corresponden a las mediciones hechas con velocidad de $1 \mathrm{seg} /$ paso dado que al medir con menor velocidad el resultado era el mismo en cuanto a intensidad y resolución de los picos, por lo cual se optó en medir con la velocidad máxima de las tres para reducir el tiempo de escaneo, dando un tiempo de 26 y 68 en intervalos de $32^{\circ}$ y $82^{\circ}$ respectivamente (Figuras 5).

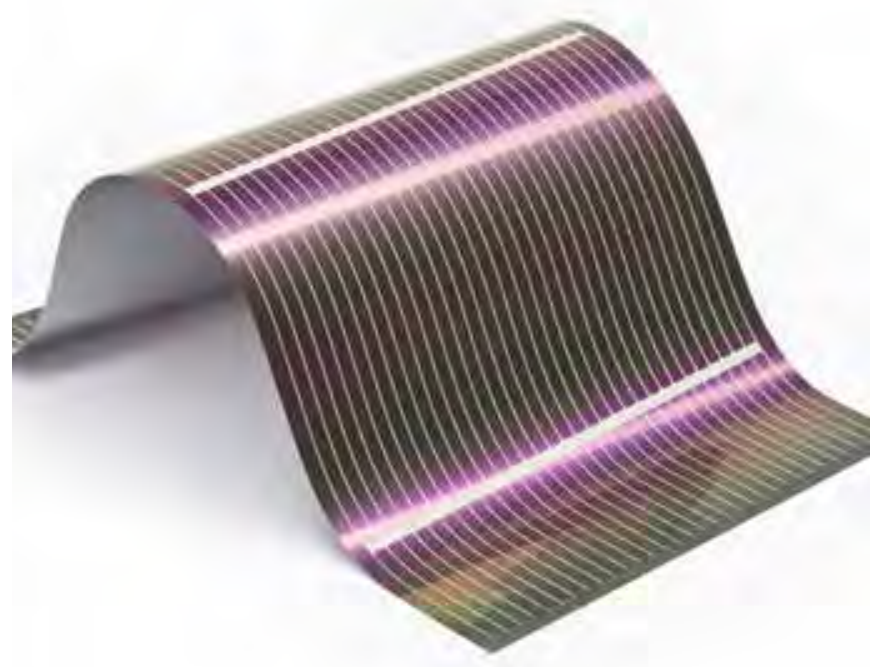

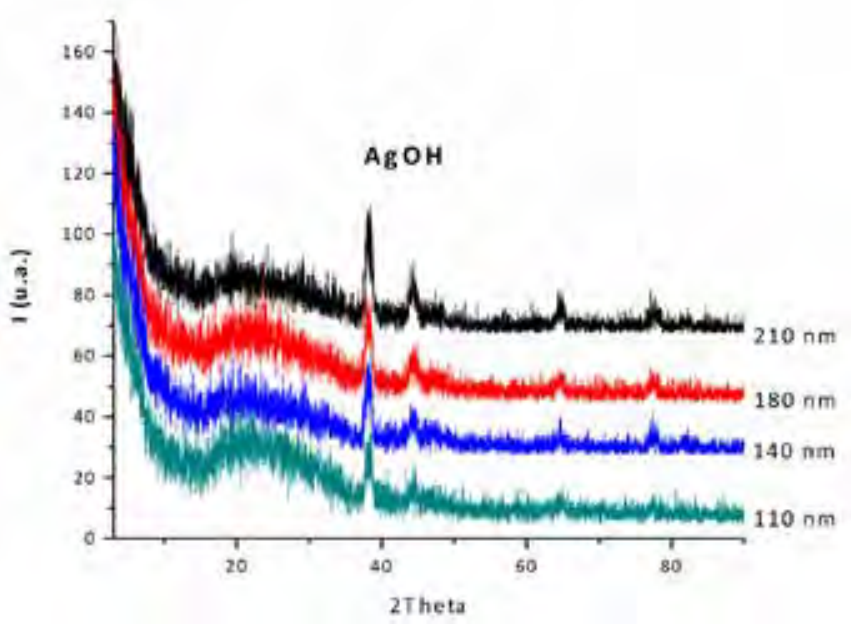
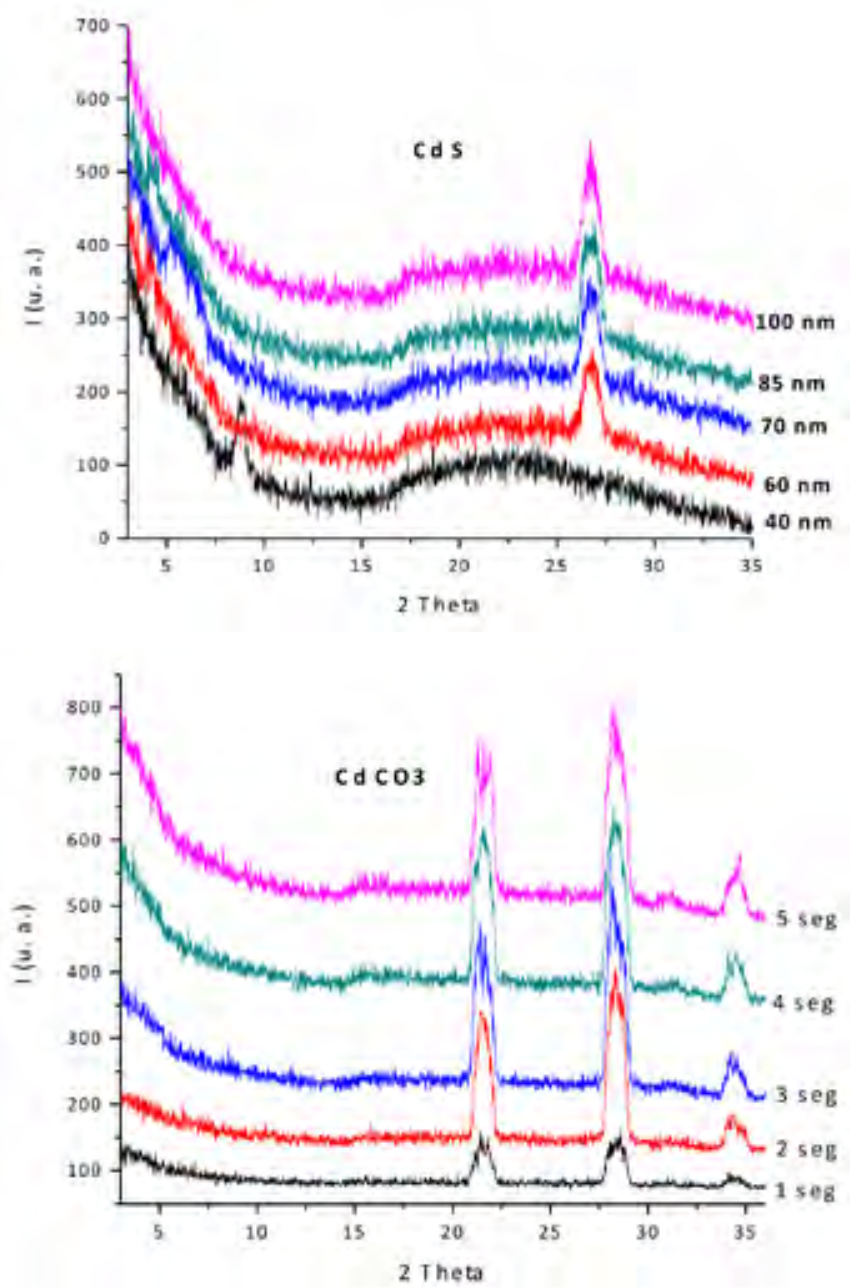

Figura 5. Los difractogramas de $\mathrm{AgOH}$ y CdS representan muestras de distintos espesores como se señala en las gráficas, mientras que en los difractogramas del $\mathrm{CdCO} 3$ representan a una sola muestra medida con diferente velocidad como es señalado. 
La integración en un mismo cuadrante, de los distintos difractogramas de $\mathrm{CdS}$ y del $\mathrm{AgOH}$, permite observar que la señal de difracción en las películas aumenta conforme aumenta el espesor, el cual depende directamente del tiempo de deposición. El incremento de la intensidad en los picos fue de 50 unidades en la película de menor espesor, y de 200 unidades en la de mayor espesor. Se confirmó la fase del CdS con la base de datos Crystallography Open Database (COD) y corresponde al patrón número 1011260 que tiene su pico principal en $2 \theta=26.5$ con índices de Miller de $(-1-1-1)$. Por otra parte, el difractograma del $\mathrm{AgOH}$ corresponde al patón número 1509146 y su pico principal se ubica en $2 \theta=38.26$ con índices de Miller de $(-1-1-1)$.

Las mediciones hechas en $\mathrm{CdCO}_{3}$ se realizaron sobre una muestra ya que el espesor es de una micra, para este compuesto la variable fue la velocidad de escaneo (de 1 a $5 \mathrm{seg} /$ paso) cuanto mayor sea el tiempo de escaneo por paso, el tiempo de medición es mayor y por lo tanto el equipo obtiene un mayor conteo que se manifiesta en el incremento de la intensidad, en este caso el incremento fue desde 50 unidades en el escaneo más rápido, hasta 350 unidades en el escaneo más lento. Sin embargo, la velocidad de escaneo no es el factor determinante como ya se vio en el caso del $\mathrm{CdS}$ y el $\mathrm{AgOH}$, el incremento de intensidad también depende de la cristalinidad del material y del espesor de la película. La fase correspondiente al $\mathrm{CdCO}_{3}$ fue corroborada con el patrón número 10111341 y su pico principal se ubica en $2 \Theta=30.4$ con índices de Miller de $(-2-1-1)$.

Al comparar los difractogramas obtenidos en este trabajo, con difractogramas en los que se ha utilizado el espejo Gobel [4], la diferencia es muy clara en cuanto a la resolución de la gráfica, dicha resolución tiene que ver con la configuración y el ancho del pico, la configuración del pico puede ser hasta cierto punto irrelevante, ya que lo más importante es la ubicación del pico en el ángulo $2 \Theta$ correspondiente. Sin embargo, el ancho del pico es algo crítico cuando el objetivo es conocer el tamaño de la celda unitaria, ya que ésta se determina con la ecuación de Scherrer; $D=K \lambda / \beta \cos \theta$, donde $\beta$ es el ancho del pico $[5,6]$.

\section{CONCLUSIONES}

Los resultados obtenidos sugieren la viabilidad de emplear un difractómetro convencional de polvos para la medición de películas delgadas mayormente cuando el espesor excede de los $100 \mathrm{~nm}$.

Se pueden mejorar los resultados de los difractogramas haciendo más pruebas con diferentes condiciones de trabajo del instrumento, ya que como se ha visto en este trabajo, en algunos materiales el tiempo de escaneo puede llegar a ser un factor para obtener un mejor difractograma.

Con las mediciones hachas con un difractómetro convencional, se observa un ancho mayor de los picos en comparación con el ancho de los picos de materiales que han sido medidos en instrumentos equipados con espejo
Gobel, esto es algo crítico para determinar el tamaño de la celda unitaria, la cual se obtiene con la ecuación de Scherrer; $D=K \lambda / \beta \cos \theta$, ya que $\beta$ es el ancho del pico.

\section{BIBLIOGRAFÍA}

[1] L. García, A. M. Courrech, L. Zamora, A. López, T. Hernández, y J. Hernández. Recubrimientos de HfN y AlHfN fabricados por Sputtering. Memorias del XIX Congreso Internacional Anual de la SOMIM: 890, 2013.

[2] M. Marciszko. A. Baczamanski, K. Wierzbanowski, M. Wróbel, C. Braham, J. P. Chopart, A. Lodini, J.Bonarski, N. Zazi. Application of multireflection grazing incidence method for stress measurements in polished Al-Mg ALLOY AND CrN coating. Applied Surface Science 266:256-267, 2013.

[3] M. B. Orduño. Síntesis y caracterización de películas delgadas de sulfuro de cadmio (CdS), preparadas bajo condición variable de reacción el método de deposición en baño químico (DbQ). Tesis profesional. Químico Biólogo, Univ. Sonora, pp. 22-27, 1998.

[4] O. Toma, L. Ion, S. Iftimie, A. Radu, S. Antohe. Structural, morphological and optical properties of rf-Sputtered CdS thin films. Material and Design 100: 198-203, 2016.

[5] P. Scherrer, Göttinger Nachrichten Gesell: Vol. 2, p 98, 1918.

[6] J. M. Sasaki. Acta Crystallographica A-Foundation and Advances: Vol. 72, 385-390, 2016

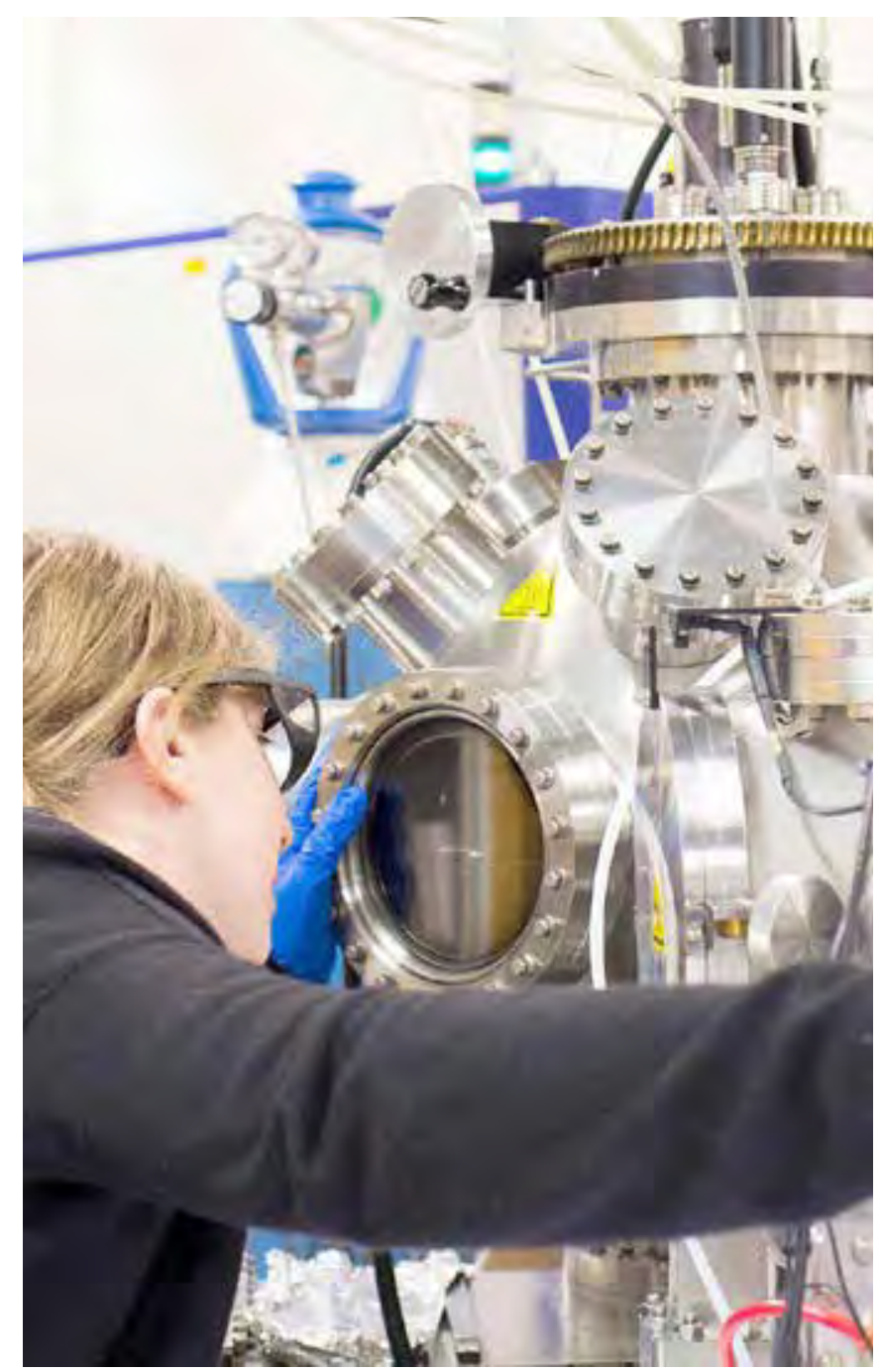

